\title{
An ERP investigation of the co-development of hemispheric lateralization of face and word recognition
}

\author{
Eva M. Dundas*, David C. Plaut, Marlene Behrmann \\ Department of Psychology, Carnegie Mellon University, Pittsburgh, PA 15213-3890, USA
}

\section{A R T I C L E I N F O}

\section{Article history:}

Received 14 July 2013

Received in revised form

3 April 2014

Accepted 13 May 2014

Available online 13 June 2014

Keywords:

Hemispheric specialization

Lateralization

Face processing

Word processing

Event-related potentials

\begin{abstract}
A B S T R A C T
The adult human brain would appear to have specialized and independent neural systems for the visual processing of words and faces. Extensive evidence has demonstrated greater selectivity for written words in the left over right hemisphere, and, conversely, greater selectivity for faces in the right over left hemisphere. This study examines the emergence of these complementary neural profiles, as well as the possible relationship between them. Using behavioral and neurophysiological measures, in adults, we observed the standard finding of greater accuracy and a larger N170 ERP component in the left over right hemisphere for words, and conversely, greater accuracy and a larger N170 in the right over the left hemisphere for faces. We also found that although children aged 7-12 years revealed the adult hemispheric pattern for words, they showed neither a behavioral nor a neural hemispheric superiority for faces. Of particular interest, the magnitude of their N170 for faces in the right hemisphere was related to that of the N170 for words in their left hemisphere. These findings suggest that the hemispheric organization of face recognition and of word recognition does not develop independently, and that word lateralization may precede and drive later face lateralization. A theoretical account for the findings, in which competition for visual representations unfolds over the course of development, is discussed.
\end{abstract}

(c) 2014 Elsevier Ltd. All rights reserved.

\section{Introduction}

The adult human brain appears to have highly specialized and seemingly independent neural systems for the visual processing of words and faces. Extensive evidence has demonstrated that the processing network for visual word recognition shows greater selectivity in the left over the right hemisphere, and, conversely, the processing network for face recognition shows greater selectivity in the right over the left hemisphere (for some early examples, see, e.g., Cohen and Dehaene (2004) and Kanwisher, McDermott, and Chun (1997)). The key issue addressed by the current work concerns the developmental emergence of this mature profile of lateralization and the degree of independence of these complementary patterns of hemispheric lateralization. Behavioral and neurophysiological data are presented that support a theoretical view in which the emerging hemispheric category-selectivity for word and face recognition are tightly coupled and are not independent of each other.

\footnotetext{
* Corresponding author. Tel.: +1 412268 8112; fax: +1 4122682798 .

E-mail addresses: edundas@andrew.cmu.edu (E.M. Dundas), plaut@cmu.edu (D.C. Plaut), behrmann@cmu.edu (M. Behrmann).
}

\subsection{Emergence of word specificity}

The dominance of the left over the right hemisphere for visual word processing is well established in right-handed adults (Grüsser \& Landis, 1991; Hellige, Laeng, \& Michimata, 2010). This left hemisphere (LH) superiority for words has long been demonstrated in behavioral studies, with participants showing an advantage for identifying orthographic stimuli presented in the right visual field (RVF) over those presented in the left visual field (LVF). Consistent with this, neurophysiological studies using eventrelated potentials (ERPs) have observed a N170 component that is stronger in the $\mathrm{LH}$ than in the $\mathrm{RH}$ in response to visually presented words (Maurer, Rossion, \& McCandliss, 2008; Mercure, Cohen Kadosh, \& Johnson, 2011). Additionally, neuroimaging studies have identified a region of the inferior temporal cortex, the Visual Word Form Area (VWFA; Talairach coordinates: $x=-43$, $y=-54, z=-12$ ), that shows greater selectivity for words over other visual stimuli in the left than right hemisphere (Cohen et al. 2000; Puce, Allison, Asgari, Gore, \& McCarthy, 1996; for review, see Price \& Devlin, 2011).

This selectivity for orthographic over non-orthographic stimuli is not present in pre-literate children and becomes apparent only after reading instruction and practice (Maurer, Brandeis, \& McCandliss, 2005; Maurer, Brem, Bucher, \& Brandeis, 2005). The left lateralization 
increases with reading ability (Marcel, Katz, \& Smith, 1974; Shaywitz et al., 2002) and the N170 continues to strengthen through adolescence (Posner \& McCandliss, 1999; Schlaggar et al., 2002). This experience-dependent neural signature can also be observed in adulthood as the increased selectivity for learned orthography is seen functionally in adults learning to read a second language (Baker et al., 2007) and structurally in adults learning to read a first language (Carreiras et al., 2009).

\subsection{Emergence of face specificity}

Unlike with written words, children have extensive experience with processing faces right from birth and, given the social importance of faces, one might expect that there would be substantial evolutionary pressure to acquire early competence in face perception. Surprisingly, then, face selectivity follows the same prolonged developmental trajectory as words. Neuroimaging studies have identified a region in right inferior temporal cortex of adults that shows greater activation to upright compared with other non-face objects. This region has been referred to as the "Fusiform Face Area" (FFA; Talairach coordinates: $x=40, y=-55$, $z=-10$ ), (Kanwisher, 2000; Kanwisher et al., 1997; Sergent \& Signoret, 1992; Spiridon, Fischl, \& Kanwisher, 2006). Consistent with this, neurophysiological studies using ERPs have observed a N170 component that is stronger in the RH than in the $\mathrm{LH}$ in response to visually presented faces (Scott \& Nelson, 2006). In children, selective activation of the FFA for faces is three times smaller than that in adults (Golarai et al., 2007) and emerges slowly through childhood and adolescence (Cohen Kadosh, Cohen Kadosh, Dick, \& Johnson, 2010; Joseph, Gathers, \& Bhatt, 2011; Scherf, Behrmann, Humphreys, \& Luna, 2007). Although some face selectivity may be apparent in the right but not left fusiform gyrus as early as 4-5 years of age (faces versus shoes, Cantlon, Pinel, Dehaene, \& Pelphrey, 2010), the laterality pattern is still far from adult-like in 5-8 year olds (Scherf et al., 2007) and is not stable until early adolescence (12-14 years; Aylward et al., 2005).

The prolonged development of the neural organization of face recognition is also reflected in the protracted emergence of behavioral skill. Adult levels of performance are not achieved by 10 -year-olds when they perform identity matching of faces differing in the spacing between the features (Mondloch, Robbins, \& Maurer, 2010). Furthermore, children continue to show large improvements in their recognition of unfamiliar faces until about 12 years of age, in contrast with their adult levels of performance in recognizing unfamiliar houses (Diamond \& Carey, 1977) and shoes (Teunisse \& De Gelder, 2003).

\subsection{Coupled emergence of word and face hemispheric lateralization}

Despite the common expectation that the emergence of visual word recognition and face recognition should be independent and that selectivity (and lateralization) for faces should be evident earlier than that for words, one recent theory has argued that the emergence of $\mathrm{RH}$ specialization for faces is contingent on the prior lateralization of words in the LH (Plaut \& Behrmann, 2011; Behrmann \& Plaut, 2013). On this account, by virtue of the fact that both faces and words rely on fine-grained visual acuity, they compete for higher-level visual representation in the mid-fusiform region (Hasson, Levy, Behrmann, Hendler, \& Malach, 2002). The middle fusiform gyrus is ideally situated to optimize such representations given that it occupies the anterior extrapolation of the fovea in extrastriate cortex. Because the visual representations of words are subject to a top-down pressure to communicate with languagerelated information (particularly phonology), these orthographic representations become partially (although not exclusively) leftlateralized (hence the left hemisphere advantage for word processing). Consequently, to minimize competition for representation in the same cortical space as words (which ensues because the image statistics of faces and words are so dissimilar), faces become more (albeit not exclusively) right-lateralized. This face/word competition theory is supported by the finding that adults with no formal education in reading have heightened left-hemisphere activation to faces compared to literate controls, and that formal instruction in reading subsequently decreases the left, and increases the right, fusiform activation to faces (Dehaene et al., 2010). Similarly, young children show decreasing responses to faces in the left fusiform (VWFA) with increasing letter knowledge (Cantlon et al., 2011).

Additionally, within individual children, words become lateralized before faces, and the degree of face selectivity within an individual is predicted by standardized reading scores after regressing out quantitative reasoning scores, age, and accuracy on a face discrimination task (Dundas, Plaut, \& Behrmann, 2013). This pattern of earlier hemispheric specialization for words than for faces (and their association) was obtained by hemifield behavioral measures that required participants (children, young adolescents and adults) to match a word or face stimuli in either the LVF or RVF with a centrally presented word or face. The index of hemispheric superiority was obtained by comparing the accuracy for judging stimuli presented in the two fields. Although these half-field measures reveal hemispheric superiorities for word and face recognition, they provide only rather general evidence regarding the neural basis of the hemispheric effects.

\subsection{The current investigation}

To examine neurophysiological markers of developmentally emerging hemispheric specificity, we recorded continuous electroencephalogram (EEG) and compared event related potentials (ERPs) in a group of children and a group of adults while they performed same/different discriminations of words and of faces. In particular, we chose to focus on the N170 ERP component because in ventral occipitotemporal cortex it has been associated with learned category selectivity (Rossion, Curran, \& Gauthier, 2002). To be able to explore the developmental changes in the ERP signals, we included children across abroad range of ages (from 7 to 12 years of age). This was done deliberately so as to provide us with sufficient variability and range of word recognition competence in order to permit a correlation analysis between ERP signals and behavioral performance.

We expected to replicate our previous finding of a LH advantage for words in both groups and a RH advantage for faces in adults but not in children. If the neural correlates of face recognition and word recognition reflect the same lateralization profile, then the lateralization of the N170 component over posterior electrodes should follow a similar developmental profile, with a greater N170 response for words over the LH in both groups and a greater N170 response for faces over the RH but just for adults. Moreover, if the emergence of face lateralization is contingent on word lateralization, then the emergence of the RH N170 specificity for faces should be predicted by the specificity of the LH N170 for words. For completeness, we also explored neurophysiological signatures of face and word selectivity at other visual ERP components, the P100 and N250; however, we expected the lateralized emergence to be specific to the N170 component because of its association with category specificity.

\section{Materials and methods}

\subsection{Participants}

All participants were monolingual native English speakers and right-handed as determined by their having an index of 80 or higher (out of 100) on the Edinburgh Handedness Inventory. In the adult group, there were 17 individuals (10 males, 
7 females) whose ages ranged from 18 to 29 years (mean 22.12 years, $S D=3.92$ ). In the child group, there were 17 individuals ( 11 males, 6 females) whose ages ranged from 7.17 to 11.75 years (mean $=9.06, S D=1.26$ ). All participants had normal or corrected-to-normal vision. The adult participants were recruited from the subject pools at Carnegie Mellon University and all provided informed consent to participate. The child participants were recruited by flyers distributed to local elementary schools and by word of mouth, and parents provided informed consent to participate. Children were compensated \$25 an hour and adults were either compensated $\$ 25$ an hour or given course credit. The protocol was approved by the Institutional Review Board at Carnegie Mellon University.

\subsection{Stimuli}

Thirty male and thirty female face images obtained from the Face-Place Database Project (Copyright 2008, Dr M. Tarr, http://wiki.cnbc.cmu.edu/Face_Place) were used in this experiment. All images of faces contained a photograph of a forward directed, young adult face, with neutral expression (see example in Fig. 1). The faces were cropped to remove hair cues and presented in grayscale against a black background. Stimuli were $1.5 \mathrm{in}$. in height and $1 \mathrm{in}$. in width, yielding visual angles of $4.8^{\circ}$ and $3.2^{\circ}$, respectively. On each trial, the pair of faces matched on gender.

The word stimuli consisted of 60 four-letter words ( 30 pairs), presented in gray, Arial, 18-point font against a black background (for word list see Appendix A). Stimuli were approximately $1 / 2$ in. in height and 1 in. in width, yielding visual angles of $1.6^{\circ}$ and $3.2^{\circ}$, respectively. Pairs were matched so that the words differed by one of the interior letters; half the pairs differed in the 2nd letter and the other halfdiffered in the 3rd letter (see example in Fig. 1). The mean word frequency in English was.0002 (range $=.00001$ to .02) (Davies, 2008) and mean bigram frequency of .124 (range $=.085$ to.141) (Jones \& Mewhort, 2004). These stimuli have been used successfully to reveal the right visual field superiority for words and the left visual field superiority for faces (Dundas et al., 2013).

\subsection{Procedure}

The experiment was run on a Dell Dimension 4700 computer using E-prime software and an Iiyama vision master 1415 monitor. Participants sat approximately 24 in. from the screen and viewed a central fixation cross whose duration ranged between 1500 and $2500 \mathrm{~ms}$. Following the offset of the fixation cross, a centrally presented (word or face) stimulus appeared for $750 \mathrm{~ms}$ and was followed immediately by a second stimulus of the same class (word or face) presented for $150 \mathrm{~ms}$ in either the left or right visual field (see Fig. 1). The center of the lateralized stimulus was $5.3^{\circ}$ from fixation. Participants were instructed to keep their gaze fixated centrally throughout the experiment and to respond by pressing one of two buttons to indicate whether the second stimulus was identical to the first or not (same/different judgment). The fixation cross appeared following the button press and indicated the start of the next trial. The presentation of stimuli in the left and right visual field was randomized per subject, with equiprobable presentation of stimuli in each field within a block. For each class of stimuli, there were 192 trials, which were split into six mini-blocks to allow participants time to rest in between blocks. EEG data (details below) were collected at the same time as the participants completed this behavioral study.

\subsection{EEG recording}

Electroencephalogram scalp recordings were made from $64 \mathrm{Ag} / \mathrm{AgCl}$ sintered electrodes embedded in a fiber Quik-Cap arranged according to the 10-20 naming system. The data were collected on a Dell optiplex 360 computer using Neuroscan 4 software. Ocular artifacts were monitored by 4 additional electrodes: one above and one below the left eye and one on the outer canthus of each eye. Electrodes were also placed on the right and left mastoids with the left serving as the online reference during data acquisition. The electrical signal was recorded continuously and amplified with a band-pass filter of .1-200 $\mathrm{Hz}$ and digitized at a sampling rate of $1 \mathrm{kHz}$. Trials were rejected from analysis if there was an eye blink - 100 to $300 \mathrm{~ms}$ from stimulus onset.

\subsection{ERP analysis}

The signal was low-pass filtered at a half-amplitude cut-off at $30 \mathrm{~Hz}$, high-pass filtered at. $1 \mathrm{~Hz}$, and re-referenced to the vertex $(\mathrm{CZ})$ electrode. Epochs were baseline corrected over a $200 \mathrm{~ms}$ prestimulus interval. ERP waveforms for each subject were averaged over all the centrally presented items for each stimulus class (Words and Faces). Of note, because there was no requirement to respond to the central item (and only to the lateralized stimulus), we avoided any confound of poorer performance in the younger group than in adults.

To examine hemispheric effects, the left-hemisphere electrodes, P7, P5, and P07 were averaged for each subject to create a grand average ERP waveform for each stimulus category. The same procedure was undertaken using the corresponding right-hemisphere electrodes, P8, P6, and P08. The N170 component was analyzed by taking the average amplitude in each hemisphere, between 160 and $220 \mathrm{~ms}$ after stimulus onset, separately for each individual participant. The P100 and N250 components were also measured by taking the average amplitude between 70 and $130 \mathrm{~ms}$, and the average amplitude between 250 and $350 \mathrm{~ms}$, respectively, in both hemispheres for each individual.

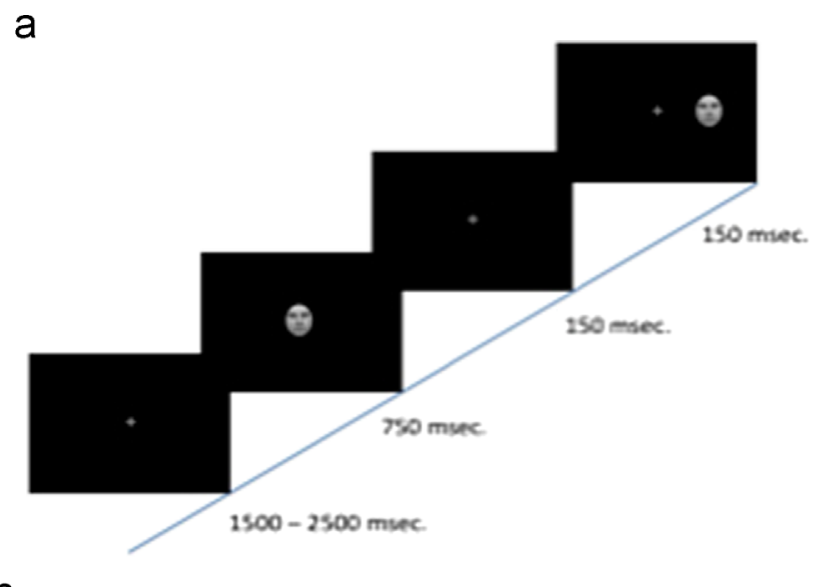

b
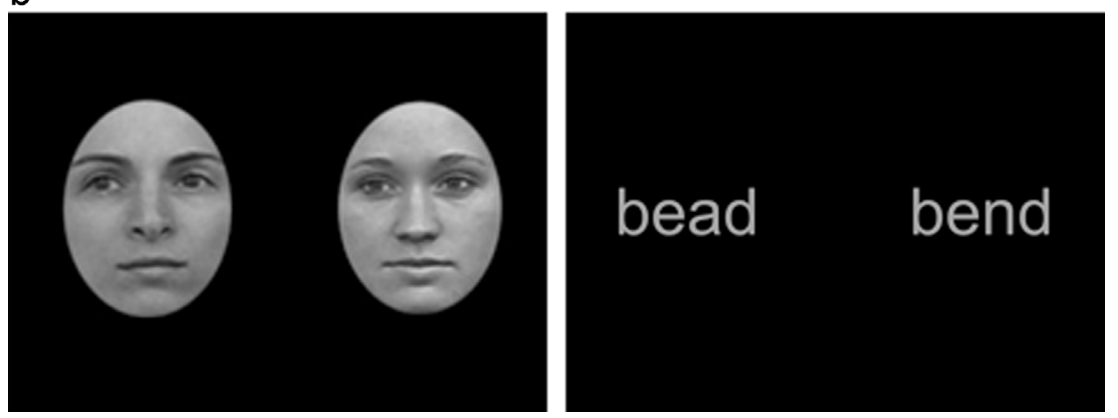

Fig. 1. (a) The temporal sequence of a single trial and (b) example pairs of word and face stimuli. 


\section{Results}

\subsection{Behavioral lateralization}

First, we investigated the lateralization of processing of words and faces as reflected by the response accuracy to stimuli presented in the LVF and RVF. Because the stimuli were presented briefly and are, thus, data limited, we report only accuracy here (also note that our previous study using this paradigm revealed no significant effects in reaction time). A $2 \times 2 \times 2$ (Word/Face Stimulus $\times$ Left/Right Visual Field as within-subjects and Child/Adult Group as between-subjects factor) ANOVA did not reveal a significant 3-way interaction $(F(1,32)=.67, p=.42)$. There was a main effect of stimulus, $(F(1,32)=5.5, p=.03)$, with higher accuracy for words than for faces (words: $M=.85$; faces: $M=.81$ ). However, there was also a group by stimulus interaction $(F(1,32)=$ 7.6, $p=.01$ ), which revealed that adults were equally accurate in responding to words and to faces (Words: $M=.83$; Faces: $M=.83 ; t$ $(16)=.39, p=.70)$ but that children performed better for words than for faces (Words: $M=.86$; Faces: $M=.78 ; t(16)=2.99, p=.01$ ). There was a significant stimulus by visual field interaction ( $F$ $(1,32)=16.77, p<.001)$ that was in the predicted direction, with higher accuracy for words in the RVF over the LVF $(t(33)=4.4$, $p<.001)$ and higher accuracy for faces in the LVF over the RVF $(t$ $(33)=2.1, p=.04)$.

Because of our specific predictions, and the stimulus by visual field interaction, we performed $2 \times 2$ (Word/Face Stimulus, Left/ Right Visual Field) ANOVAs on the adult and child data separately. A significant interaction between field and stimulus was observed for the adult group $(F(16)=22.1, p<.001)$, with higher accuracy for words in the RVF over the $\operatorname{LVF}(t(16)=3.4, p=.004)$, and for faces in the LVF over the RVF $(t(16)=3.1, p=.006$; see Fig. $2 a)$. The data for the child group revealed a trend towards a significant interaction $(F(1,16)=3.7, p=.07)$, with significantly higher accuracy for words in the RVF over the $\operatorname{LVF}(t(16)=2.4, p=.01)$, but no difference in accuracy for faces in the two fields $(t(16)=.64$ $p=.53$; see Fig. $2 \mathrm{~b}$ ).

a

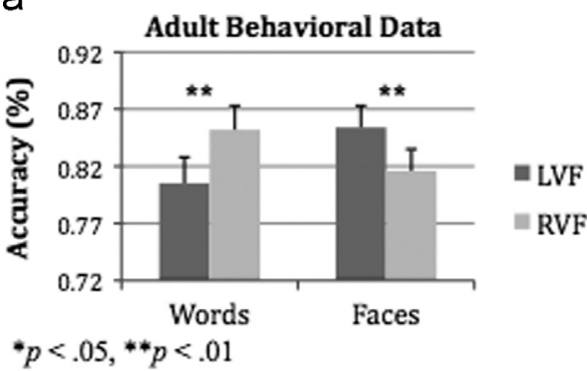

b

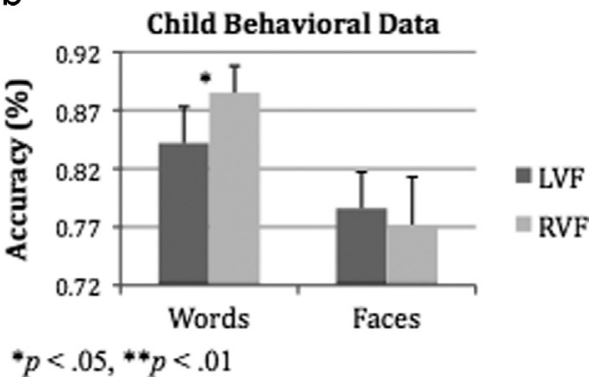

Fig. 2. (a) Mean accuracy ( $+1 \mathrm{SE}$ ) for adult group for words and faces in the left and right visual fields. (b) Mean accuracy ( $+1 S E$ ) for child group for words and faces in the left and right visual fields.

\subsection{Lateralization of N170 component}

To examine the electrophysiological correlates of word and face hemispheric lateralization, we first examined the signature N170 component in the right and left hemispheres. Using the amplitude of the N170 component as the dependent measure, a $2 \times 2 \times 2$ (Word/Face Stimulus, Right/Left Hemisphere, Adult/Child Group) ANOVA did not reveal a significant 3-way interaction $(F(1,32)=.56$, $p=.41)$. There was a significant stimulus by hemisphere interaction $(F(1,32)=10.2, p=.003)$, with more negative amplitude for words in the LH over the $\mathrm{RH}(t(33)=3.33, p=.002)$ but no difference in amplitude for faces between the two hemispheres $(t(16)=1.47, p=15)$. There was also a significant stimulus by group interaction $(F(1,32)=10.62, p=.003)$ which reflected the greater negative amplitude for faces in adults than in children $(t(32)=$ $3.45, p=.037)$.

To test our a priori predictions that hemispheric differences in word and face representation vary as a function of age, we examined the stimulus by hemisphere interaction as a function of age in a $2 \times 2$ (Word/Face Stimulus, Right/Left Hemisphere) ANOVA on the data from the adult and child groups separately. The adult group demonstrated a significant 2-way interaction between stimulus type and hemisphere $(F(1,16)=7.67, p=.014)$; consistent with the behavioral data, there was a significantly greater negative amplitude for words in the LH over the $\mathrm{RH}(t(16)$ $=2.11, p=.05$ ), and a greater negative amplitude for faces in the $\mathrm{RH}$ over the $\mathrm{LH}(t(16)=2.43, p=.027$; see Fig. $3 \mathrm{a})$. The child group also showed a significant 2-way interaction between stimulus type and hemisphere, $(F(1,16)=4.94, p=.041)$; the pattern of lateralization was again consistent with the behavioral data, with greater negative amplitude for words in the LH over the $\mathrm{RH}(t(16)=2.71$, $p=.016$ ) but no amplitude difference for faces across the hemispheres $(t(16)=.81, p=.43$; see Fig. $3 \mathrm{~b})$. These age differences for words versus faces are clearly apparent in the grand average waveforms and topographic scalp distribution of the N170 component shown Fig. $4 a-c$. As evident in these scalp maps (Fig. 4c), whereas the adults show the complementary selectivity for both stimulus types, in the children, there is greater negative amplitude in the left than right hemisphere for words and less hemispheric selectivity for faces.

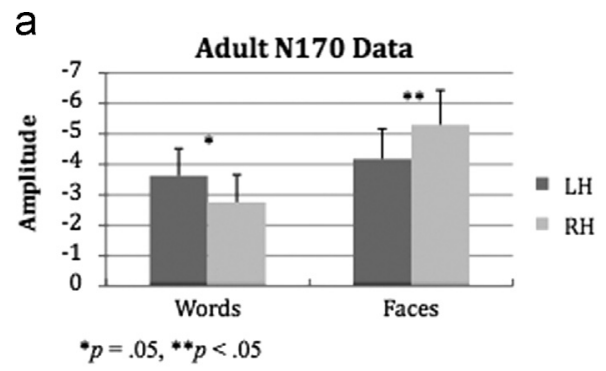

b

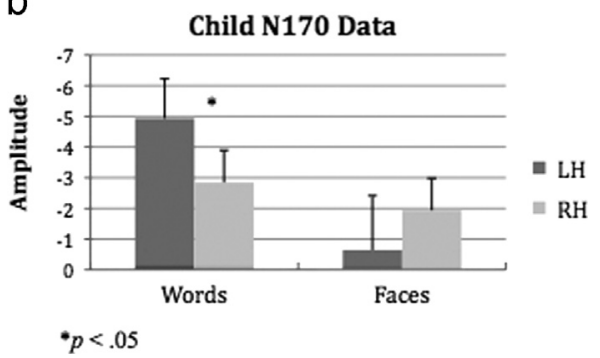

Fig. 3. (a) Mean N170 amplitude (+1SE) for adult group for words and faces in the left and right hemispheres. (b) Mean N170 amplitude (+1SE) for child group for words and faces in the left and right hemispheres. 
a

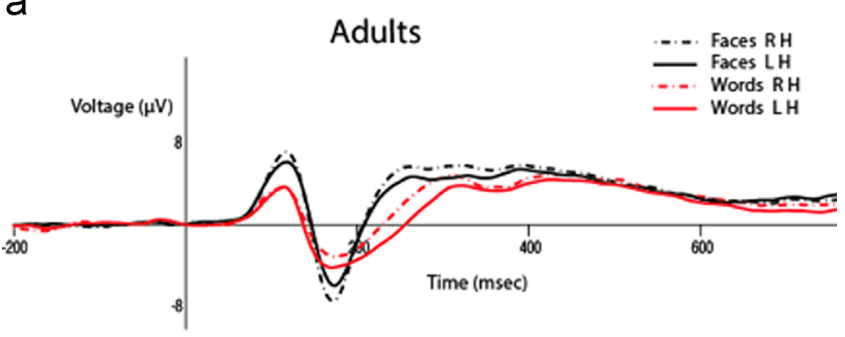

b

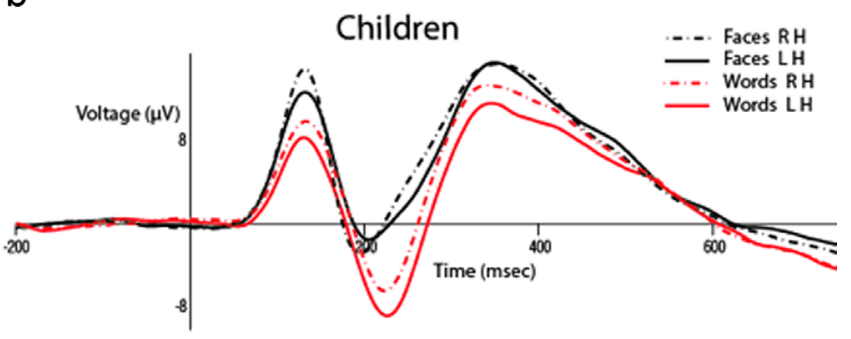

C
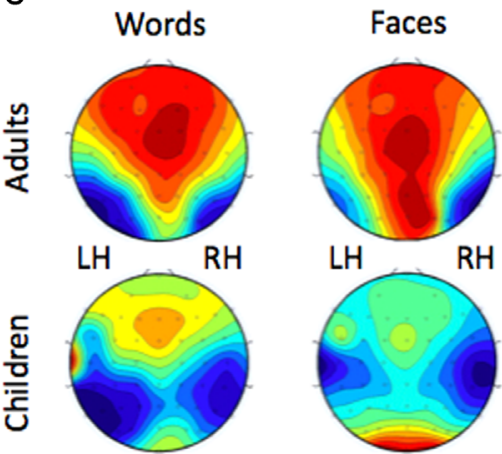

Fig. 4. (a) Adult group ERP waveforms for words and faces in the LH and the RH. (b) Child group ERP waveforms for words and faces in the LH and the RH. (c) The topographic scalp distributions of the mean amplitude between 160 and 220 ms for words and faces in both groups.

We then examined the relationship between the N170 component for words and for faces by correlating the N170 amplitude for words and for faces for each individual in both hemispheres. In adults, the amplitude of the N170 component for words was correlated across the $\mathrm{LH}$ and $\mathrm{RH}\left(r^{2}=.80, p<.001\right)$, the N170 component for faces was correlated across the $\mathrm{LH}$ and $\mathrm{RH}$ $\left(r^{2}=.84, p<.001\right)$, and the N170 components for words and faces were correlated cross both hemispheres (N170 faces RH - words $\mathrm{LH}, r^{2}=.68, p<.001$; N170 face LH - words RH, $r^{2}=.41, p=.006$; see Table 1 and Fig. 5). These same correlations were not present in the child group, with only the N170 amplitude for words being correlated across hemispheres $\left(r^{2}=.64, p<.001\right)$, and the N170 amplitude for faces across hemispheres was only approaching significance $\left(r^{2}=.19, p=.09\right)$ Interestingly, it was the amplitude for words in both hemispheres that predicted the amplitude of the $\mathrm{N} 170$ for faces in the RH (N170 words in $\mathrm{LH}-$ faces in $\mathrm{RH}, r^{2}=.32$, $p=.017$; N170 words RH - faces RH, $\left.r^{2}=.43, p=.004\right)$. The same was not true for the N170 amplitude for faces in the LH; see Table 2.

\subsection{Lateralization of the P100 and N250 components}

As a means of assessing whether this developmental pattern was specific to the N170 component, the same ANOVA as that adopted with the N170 component was conducted with the P100 and N250 components. For the P100, there was a significant main
Table 1

Correlations of N170 amplitude for words and faces in right and left hemispheres in adult group $(N=17)$.

\begin{tabular}{lllll}
\hline Variables & 1 & 2 & 3 & 4 \\
\hline 1. Words LH & - & & & \\
2. Words RH & $.801^{* *}$ & - & - & - \\
3. Faces LH & $.602^{* *}$ & $.408^{*}$ & $.837^{*}$ & \\
4. Faces RH & $.679^{* *}$ & $.466^{*}$ & & \\
\hline$* p<.10$. & & & & \\
$* p<.001$. & & & &
\end{tabular}
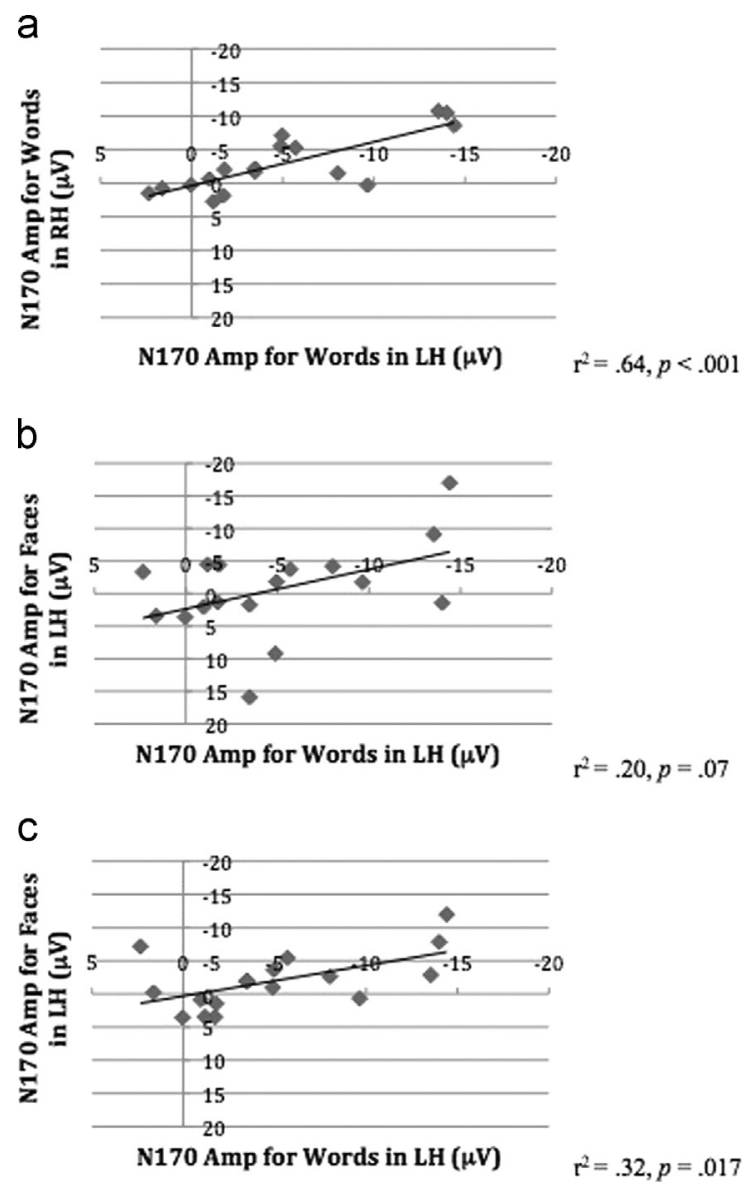

Fig. 5. (a) Scatter plot and correlation analysis showing the relationship between N170 amplitude for words in the LH and words in the RH in child group. (b) Scatter plot and correlation analysis showing the relationship between N170 amplitude for words in the LH and faces in the LH in child group. (c) Scatter plot and correlation analysis showing the relationship between N170 amplitude for words in the LH and faces in the RH in child group.

Table 2

Correlations of N170 amplitude for words and faces in right and left hemispheres in child Group $(N=17)$

\begin{tabular}{lllll}
\hline Variables & 1 & 2 & 3 & 4 \\
\hline 1. Words LH & - & & & \\
2. Words RH & $.643^{* *}$ & - & & \\
3. Faces LH & .201 & .084 & - & - \\
4. Faces RH & $.324^{*}$ & $.429^{* * *}$ & .182 & - \\
\hline
\end{tabular}

$* p<.05$
$* * *$
$*<.01$

effect of stimulus $(F(1,32)=45.6, p<.001)$, with greater amplitude for faces than words (Faces: $M=11.39$; Words: $M=3.25$ ) and a marginally significant main effect of hemisphere $(F(1,32)=3.88$, 
a

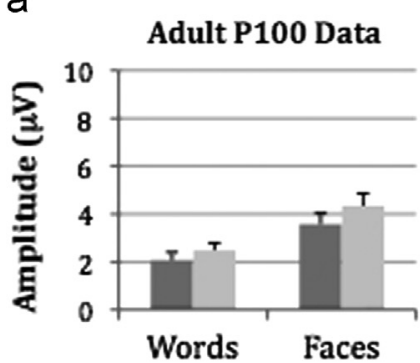

C

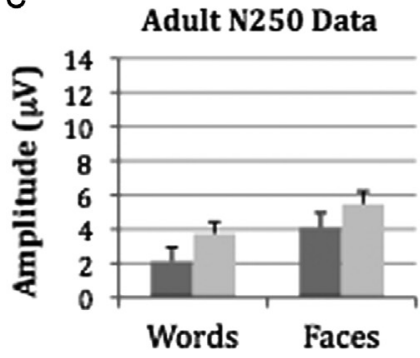

b

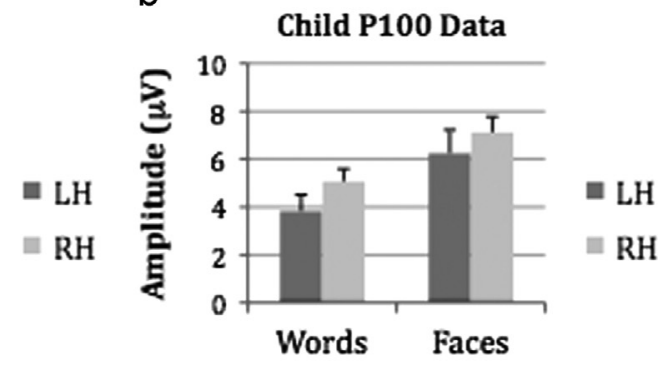

d
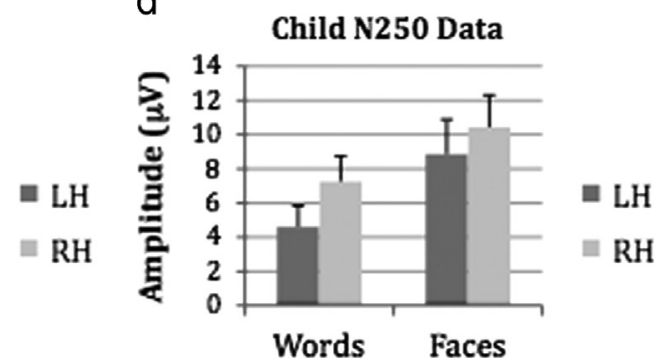

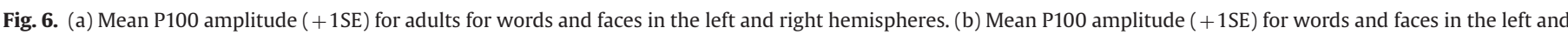

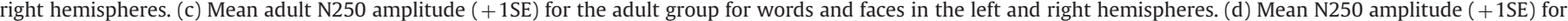
the child group for words and faces in the left and right hemispheres.

$p=.058)$, with greater amplitude in the $\mathrm{RH}$ over the LH (RH: Mean=5.33; LH: Mean=3.37). Additionally, there was a main effect of group $(F(1,32)=14.08, p=.001)$, with children having a larger P100 amplitude than adults (Children: Mean=5.57; Adults: Mean $=3.12$; see Fig. $6 a$ and $b$ ). There were no other significant effects. Furthermore, analyzing the adult and child groups separately did not reveal any significant hemisphere by stimulus interactions.

The same analysis using the N250 component revealed a significant main effect of stimulus $(F(1,32)=18.1, p<.001)$, with greater amplitude for faces than words (Faces: $M=7.28$; Words: $M=4.43)$ and a main effect of hemisphere $(F(1,32)=7.83, p=.009)$, with greater amplitude in the RH over the LH (RH: $M=6.73$, LH: $M=4.92)$. Additionally, there was a main effect of group, $(F(1,32)=$ $6.14, p=.02$ ), with children having a larger N250 amplitude than adults (Children: $M=7.79$; Adults: $M=3.86$; see Fig. $6 c$ and d). There were no other significant effects. Analyzing the adult and child groups separately did not reveal any significant hemisphere by stimulus interactions. ${ }^{2}$

\section{Discussion}

Despite intuitions that the neural organization for the visual processing of faces and words should be independent, and that hemispheric specialization for faces should precede that for words, there is now accumulating evidence for interdependence, and in particular, for the view that hemispheric specialization for face recognition emerges out of competition for higher-order visual representation with words. The current study examined the developmental emergence of hemispheric specialization, measured behaviorally and neurophysiologically, for the processing of words and of faces, and, in particular, assessed whether there

\footnotetext{
${ }^{1} 2 \times 2$ (Stimulus $\times$ Hemisphere) ANOVA with the P100 amplitude: Adults $F$ $(1,16)=1.28, p=.26$; Children $F(1,16)=1.46, p=.24$.

$22 \times 2$ (Stimulus $\times$ Hemisphere) ANOVA with the N250 amplitude: Adults $F$ $(1,16)=.18, p=.68$; Children $F(1,16)=1.24, p=.28$.
}

was any association between the representations in these two domains.

In adults, we replicated the standard behavioral finding of significantly higher accuracy for words in the LH than the $\mathrm{RH}$, and conversely, significantly higher accuracy for faces in the RH than the LH. As with our previous study (Dundas et al., 2013), the accuracy advantage for words and for faces under lateralized presentation was equally, and oppositely matched, ensuring that this paradigm is well suited to examine possible hemispheric biases in the children. The pattern of data obtained from the children mirrored our previous result in which children were more accurate at recognizing words presented to the $\mathrm{LH}$ over the $\mathrm{RH}$, but exhibited no hemispheric difference in accuracy for recognizing faces (Dundas et al., 2013). Although adults performed equally well for words and faces, children performed better on words than they did on faces.

The pattern of behavioral advantage across groups was also reflected in the lateralization profiles of the N170 elicited in response to the centrally presented words and to faces. Adults demonstrated the standard finding of greater negative amplitude of the N170 component for words in the $\mathrm{LH}$ over the $\mathrm{RH}$, and conversely, greater negative amplitude of the $\mathrm{N} 170$ component for faces in the $\mathrm{RH}$ over the $\mathrm{LH}$. Children also demonstrated greater negative amplitude of the N170 component for words in the LH over the RH. This pattern is consistent with data showing that the lateralization of the N170 component for words emerges shortly after children learn how to read (Maurer, Brem, Bucher, \& Brandeis, 2005). Consistent with the absence of a hemispheric advantage for face recognition in behavior, the children did not evince a hemispheric difference in the amplitude of the N170 component for faces.

For adults, the amplitude of the N170 component for words and for faces, in both hemispheres, were all correlated with each other. This suggests that in adults, who have mature and stable neural organization, the tuning of the neural response to words in both hemispheres is related to the neural tuning of faces in both hemispheres. This finding is consistent with the literature showing a relationship between the neural organization of word and of face networks (Plaut \& Behrmann 2011; Cantlon et al., 2011; Dehaene et al., 2010; Dundas et al., 2013). In contrast to the adults, for the children, the N170 amplitude for faces in the RH was correlated 
with the N170 amplitude for words in both hemispheres but not with the N170 amplitude for faces in the LH. Thus, it appears that, over development, the tuning of the N170 for faces is driven by the tuning of the N170 for word representations. This further suggests that while the networks are developing and maturing, the LH organization for word processing is the driving force behind the $\mathrm{RH}$ organization for face processing.

Although our prediction was that the hemisphere effects would only be evident in the $\mathrm{N} 170$, for completeness we also examined the P100 and N250 components for words and for faces. Both groups showed greater amplitude for faces than words on both the P100 and N250, and children generally showed greater amplitude for both stimuli than adults did. The pattern of hemispheric specialization seen in the behavioral profile and in the N170 components was not reflected in either the P100 or N250 components for words or for faces.

Our finding that the emergence of hemispheric specialization for face selectivity is dependent on the emergence of lateralization of word selectivity is consistent with recent data showing that these two domains are not independent. For example, as children gain letter knowledge, there is a concomitant decrease in the selectivity of faces in the LH (Cantlon et al., 2011). This same competitive effect is observed in pre-literate adults who, after receiving reading instruction, show a decrease in selectivity of faces in the LH (Dehaene et al., 2010). Additionally, in a recent paper, Li et al. (2013) found that acquiring knowledge of Chinese written characters hinders the emergence of right lateralization of faces. Although, on the surface, this might seem counter to the theory we have proposed in which refinement of letter/word knowledge results in the lateralization of word processing to the $\mathrm{LH}$ and consequent lateralization of faces to the RH (Dundas et al., 2013), we would expect that the different computational constraints of Chinese and English orthography may well shift the development of faces in different ways. Thus, while we (and others) have argued that it is the reliance on phonology that triggers the word processing to become left lateralized, this pressure might not be as strong for Chinese reading. Chinese character reading may not automatically involve strong phonological processing and, perhaps as a result, the LH advantage for orthographic input is reduced in Chinese readers relative to English readers (Hull \& Vaid, 2007), and the Chinese readers show greater bilateral hemispheric activation when reading Chinese script (Liu, Dunlap, Fiez, \& Perfetti, 2007). Despite these differences, both studies support the theory that the development of mechanisms underlying face perception is influenced by experience with reading.

Our proposed explanation for this dependent relationship between words and faces (Plaut \& Behrmann, 2013) is based on the insight that both words and faces place distinctive demands on high-acuity vision. Consequently, words and faces compete for representational space in both hemispheres, specifically in the cortical region adjacent to regions of retinotopic cortex encoding information from central vision with maximal discriminability (Hasson et al., 2002; Levy, Hasson, Avidan, Hendler, \& Malach, 2001), notably the VWFA and the FFA. To minimize connection length (and the opportunity for errors to arise as signal propagation distance increases or inter-hemispheric engagement is necessary), orthographic representations are further constrained to be proximal to language-related information (especially phonological information), which is left-lateralized in most individuals. As a result, words (and, presumably, letters before that) gradually come to rely most heavily, albeit not exclusively (see right hemisphere accuracy for word discrimination in Fig. 2), on the left fusiform region (VWFA) as an intermediate cortical region bridging between early vision and language. This idea is also consistent with the interactive view that left occipitotemporal regions become specialized for word processing as a result of top-down predictions from the language system integrating with bottom-up visual inputs (Devlin, Jamison, Gonnerman, \& Matthews, 2006; Kherif, Josse, \& Price, 2011; Price \& Devlin, 2011; Twomey, Kawabata Duncan, Price, \& Devlin, 2011).

This account is further supported by recent evidence that shows that, as a group, left-handers have more bilateral face selectivity in the fusiform gyrus (Bukowski, Dricot, Hanseeuw, \& Rossion, 2013). This investigation of face lateralization revealed that, across individual left-handers, the distribution of hemispheric selectivity for faces is heterogeneous. When considering that language lateralization in left-handers is also far more heterogeneous than in right-handers, consequent heterogeneous face lateralization fits with the model of competition between words and faces. Moreover, the heterogeneity is seen only for face processing in the fusiform gyrus, which is in direct competition with words, and not in any other face-selective cortical area.

\section{Conclusions}

Our data support the claim that hemispheric specialization for visual words emerges prior to hemispheric specialization for faces, and that the neural tuning of face selectivity is driven by the neural tuning of word selectivity. These results fit well with a theory in which word processing becomes left lateralized because of the pressure to be proximal to language areas, and that, subsequently, by means of competition for representational space in the left occipitotemporal cortex, face processing becomes lateralized to the right hemisphere homolog. Despite the fact that faces and words appear so intuitively different, an interactive account of the development of word and face specialization becomes far more plausible when considering the similar computational constraints to which they are subject. This exploration into the development of hemispheric specialization for both words and faces reveals that the mechanisms giving rise to these adult patterns of lateralization are not as independent as commonly thought and that increasing literacy is the key pressure that triggers the emergence of lateralized hemispheric specialization of face processing.

\section{Acknowledgments}

This research was supported by a training grant from the National Institutes of Health (B-squared) to ED (T32GM081760), a grant from the National Science Foundation to $\mathrm{MB}$ and $\mathrm{DP}$ (BCS0923763) and a grant to MB from the NSF Science of Learning Center No. SMA-1041755 to the Temporal Dynamics of Learning Center). The authors thank the administrators and parents of the Community Day School in Pittsburgh and The Carnegie Mellon Institute for Talented Elementary and Secondary Students for their participation. We also thank Ryan Egan for his assistance with data collection and Annie Chen with her help with data processing.

\section{Appendix A}

Stimulus word pairs

$\begin{array}{ll}\text { band } & \text { bald } \\ \text { bead } & \text { bend } \\ \text { beds } & \text { buds } \\ \text { come } & \text { came } \\ \text { cord } & \text { cold } \\ \text { dorm } & \text { doom } \\ \text { foal } & \text { fowl } \\ \text { fold } & \text { ford }\end{array}$




\begin{tabular}{ll} 
head & herd \\
hook & honk \\
jobs & jabs \\
lark & lurk \\
lump & limp \\
mild & mold \\
part & pant \\
port & part \\
posh & push \\
prep & peep \\
riot & rift \\
rose & rise \\
snap & snip \\
sole & sore \\
soup & soap \\
tack & tank \\
tile & tire \\
told & toad \\
tore & tire \\
torn & turn \\
welt & wilt \\
wife & wile \\
\hline
\end{tabular}

\section{References}

Aylward, E. H., Park, J. E., Field, K. M. Parsons, A. C, Richards, T. L., Cramer, S. C., et al (2005). Brain activation during face perception: evidence of a developmental change. Journal of Cognitive Neuroscience, 17(2), 308-319, http://dx.doi.org/ $10.1162 / 0898929053124884$

Baker, C. I., Liu, J., Wald, L. L., Kwong, K. K., Benner, T., \& Kanwisher, N. (2007). Visual word processing and experiential origins of functional selectivity in human extrastriate cortex. Proceedings of the National Academy of Sciences of the United States of America, 104(21), 9087-9092, http://dx.doi.org/10.1073/ pnas.0703300104.

Behrmann, M., \& Plaut, D. C. (2013). Distributed circuits, not circumscribed centers, mediate visual recognition. Trends in cognitive sciences, 17(5), 210-219.

Bukowski, H., Dricot, L., Hanseeuw, B., \& Rossion, B. (2013). Cerebral lateralization of face-sensitive areas in left-handers: only the FFA does not get it right. Cortex, 49 (9), 2583-2589.

Cantlon, J. F., Pinel, P., Dehaene, S., \& Pelphrey, K. A. (2011). Cortical representations of symbols, objects, and faces are pruned back during early childhood. Cerebral Cortex, 21, 191-199, http://dx.doi.org/10.1093/cercor/bhq078.

Carreiras, M., Seghier, M. L., Baquero, S., Estévez, A., Lozano, A., Devlin, J. T., et al. (2009). An anatomical signature for literacy. Nature, 461(7266), 983-986, http: //dx.doi.org/10.1038/nature08461.

Cohen Kadosh, K., Cohen Kadosh, R., Dick, F., \& Johnson, M. H. (2010). Developmental changes in effective connectivity in the emerging core face network. Cerebral Cortex, 22(5), 903-917, http://dx.doi.org/10.1093/cercor/bhq215.

Cohen, L, Dehaene, S., Naccache, L., Lehéricy, S., Dehaene-Lambertz, G., Hénaff, M. A., et al. (2000). The visual word form area: spatial and temporal characterization of an initial stage of reading in normal subjects and posterior split-brain patients. Brain, 123(2), 291-307.

Cohen, Laurent, \& Dehaene, S. (2004). Specialization within the ventral stream: The case for the visual word form area. Neurolmage, 22(1), 466-476, http://dx.doi. org/10.1016/j.neuroimage.2003.12.049.

Davies, M. (2008). The Corpus of Contemporary American English: 450 million words, 1900-present. Available online at: 〈http://corpus.byu.edu/coca/ $\rangle$.

Dehaene, S., Pegado, F., Braga, L. W., Ventura, P., Nunes Filho, G., Jobert, A., et al. (2010). How learning to read changes the cortical networks for vision and language. Science, 330(6009), 1359-1364, http://dx.doi.org/10.1126/science. 1194140.

Devlin, J., Jamison, H., Gonnerman, L., \& Matthews, P. (2006). The role of the posterior fusiform gyrus in reading. Journal of Cognitive Neuroscience, 18(6), 911-922.

Diamond, R., \& Carey, S. (1977). Developmental changes in the representation of faces. Journal of Experimental Child Psychology, 23(1), 1-22.

Dundas, E. M., Plaut, D. C., \& Behrmann, M. (2013). The joint development of hemispheric lateralization for words and faces. Journal of Experimental Psychology: General, 142(2), 348.

Golarai, G., Ghahremani, D. G., Whitfield-Gabrieli, S., Reiss, A., Eberhardt, J. L., Gabrieli, J. D. E., et al. (2007). Differential development of high-level visual cortex correlates with category-specific recognition memory. Nature Neuroscience, 10(4), 512-522, http://dx.doi.org/10.1038/nn1865.

Grüsser, O. J., \& Landis, T. (1991). The splitting of "I" and "me": heautoscopy and related phenomena. Visual agnosias and other disturbances of visual perception and cognition (pp. 297-303)Amsterdam: MacMillian.
Hasson, U., Levy, I., Behrmann, M., Hendler, T., \& Malach, R. (2002). Eccentricity bias as an organizing principle for human high-order object areas. Neuron, 34(3), 479-490 (Retrieved from)/http://www.ncbi.nlm.nih.gov/pubmed/ $11988177\rangle$.

Hellige, J. B., Laeng, B., \& Michimata, C. (2010). Processing asymmetries in the visual system. In: R. Hugdahl, \& K. Westerhausen (Eds.), The two halves of the brain: information processing in the cerebral hemispheres (pp. 379-415). Cambridge, MA: MIT Press.

Hull, R., \& Vaid, J. (2007). Bilingual language lateralization: a meta-analytic tale of two hemispheres. Neuropsychologia, 45(9), 1987-2008, http://dx.doi.org/ 10.1016/j.neuropsychologia.2007.03.002.

Jones, M. N., \& Mewhort, D. J. K. (2004). Case-sensitive letter and bigram frequency counts from large-scale English corpora. Behavior Research Methods, Instruments, \& Computers, 46(3), 388-396.

Joseph, J. E., Gathers, A. D., \& Bhatt, R. S. (2011). Progressive and regressive developmental changes in neural substrates for face processing: testing specific predictions of the interactive specialization account. Developmental Science, 14 (2), 227-241, http://dx.doi.org/10.1111/j.1467-7687.2010.00963.x.

Kanwisher, N. (2000). Domain specificity in face perception. Nature Neuroscience, 3 (8), 759-763.

Kanwisher, N., Mcdermott, J., \& Chun, M. M. (1997). The fusiform face area: a module in human extrastriate cortex specialized for face perception. NeuroImage, 17(11), 4302-4311.

Kherif, F., Josse, G., \& Price, C. J. (2011). Automatic top-down processing explains common left occipito-temporal responses to visual words and objects. Cerebral Cortex, 21(1), 103-114.

Levy, I., Hasson, U., Avidan, G., Hendler, T., \& Malach, R. (2001). Center-periphery organization of human object areas. Nature Neuroscience, 4(5), 533-539, http: //dx.doi.org/10.1038/87490.

Li, S., Lee, K., Zhao, J., Yang, Z., He, S., \& Weng, X. (2013). Neural competition as a developmental process: early hemispheric specialization for word processing delays specialization for face processing. Neuropsychologia, 51(5), 950-959, http://dx.doi.org/10.1016/j.neuropsychologia.2013.02.006.

Liu, Y., Dunlap, S., Fiez, J., \& Perfetti, C. (2007). Evidence for neural accommodation to a writing system following learning. Human Brain Mapping, 28(11), 1223-1234, http://dx.doi.org/10.1002/hbm.20356.

Marcel, T., Katz, L., \& Smith, M. (1974). Laterality and reading proficiency. Neuropsychologia, 12(1), 131-139 (Retrieved from)/http://www.ncbi.nlm.nih. gov/pubmed/4821184>.

Maurer, U., Brandeis, D., \& McCandliss, B. D. (2005). Fast, visual specialization for reading in English revealed by the topography of the N170 ERP response. Behavioral and Brain Functions, 1, 13, http://dx.doi.org/10.1186/1744-9081-1-13.

Maurer, U., Brem, S., Bucher, K., \& Brandeis, D. (2005). Emerging neurophysiologica specialization for letter strings. Journal of Cognitive Neuroscience, 17(10), $1532-1552$.

Maurer, U., Rossion, B., \& McCandliss, B. D. (2008). Category specificity in early perception: face and word $n 170$ responses differ in both lateralization and habituation properties. Frontiers in Human Neuroscience, 2, 1-7, http://dx.doi. org/10.3389/neuro.09.018.2008.

Mercure, E., Cohen Kadosh, K., \& Johnson, M. H. (2011). The n170 shows differential repetition effects for faces, objects, and orthographic stimuli. Frontiers in Human Neuroscience, 5(6), 1-10, http://dx.doi.org/10.3389/fnhum.2011.00006.

Mondloch, C. J., Robbins, R., \& Maurer, D. (2010). Discrimination of facial features by adults, 10-year-olds, and cataract-reversal patients. Perception, 39, 184-195, http://dx.doi.org/10.1068/p6153.

Plaut, D. C., \& Behrmann, M. (2011). Complementary neural representations for faces and words: A computational exploration. Cognitive neuropsychology, 28(34), 251-275.

Posner, M. I., \& McCandliss, B. D. (1999). Brain circuitry during reading. In: P. Klein, \& R. McMullen (Eds.), Converging methods for understanding reading and dyslexia (pp. 305-338). Cambridge, MA: MIT Press.

Price, C. J., \& Devlin, J. T. (2011). The interactive account of ventral occipitotemporal contributions to reading. Trends in Cognitive Sciences, 15(6), 246-253, http://dx. doi.org/10.1016/j.tics.2011.04.001.

Puce, A., Allison, T., Asgari, M., Gore, J. C., \& McCarthy, G. (1996). Differential sensitivity of human visual cortex to faces, letterstrings, and textures: functional magnetic resonance imaging study. The Journal of Neuroscience, 16 (16), 5205-5215 (Retrieved from).

Rossion, B., Curran, T., \& Gauthier, I. (2002). A defense of the subordinate-level expertise account for the N170 component. Cognition, 85(2), 189-196 (Retrieved from)/http://www.ncbi.nlm.nih.gov/pubmed/12127699〉.

Scherf, K. S., Behrmann, M., Humphreys, K., \& Luna, B. (2007). Visual categoryselectivity for faces, places and objects emerges along different developmental trajectories. Developmental Science, 10(4), F15-F30, http://dx.doi.org/10.1111/ j.1467-7687.2007.00595.x.

Schlaggar, B. L., Brown, T. T., Lugar, H. M., Visscher, K. M., Miezin, F. M., \& Petersen, S. E. (2002). Functional neuroanatomical differences between adults and schoolage children in the processing of single words. Science, 296(5572), 1476-1479.

Scott, L. S., \& Nelson, C. a. (2006). Featural and configural face processing in adults and infants: A behavioral and electrophysiological investigation. Perception, 35 (8), 1107-1128, http://dx.doi.org/10.1068/p5493.

Sergent, J, \& Signoret, J. L. (1992). Functional and anatomical decomposition of face processing: evidence from prosopagnosia and PET study of normal subjects. Philosophical transactions of the Royal Society of London. Series B, Biological Sciences, 335(1273), 55-61, http://dx.doi.org/10.1098/rstb.1992.0007 (discussion 61-2). 
Shaywitz, B. A., Shaywitz, S. E., Pugh, K. R., Mencl, W. E., Fulbright, R. K., Skudlarski, P., \& Gore, J. C. (2002). Disruption of posterior brain systems for reading in children with developmental dyslexia. Biological psychiatry, 52(2), 101-110.

Spiridon, M., Fischl, B., \& Kanwisher, N. (2006). Location and spatial profile of category-specific regions in human extrastriate cortex. Human Brain Mapping. 27, 77-89, http://dx.doi.org/10.1002/hbm.20169.
Teunisse, J., \& De Gelder, B. (2003). Face processing in adolescents with autistic disorder: The inversion and composite effects. Brain and Cognition, 52, 285-294, http://dx.doi.org/10.1016/S0278-2626(03)00042-3.

Twomey, T., Kawabata Duncan, K. J., Price, C. J., \& Devlin, J. T. (2011). Top-down modulation of ventral occipito-temporal responses during visual word recognition. Neuroimage, 55(3), 1242-1251. 